\title{
Literatur. $\left.{ }^{1}\right)$
}

A. Huber, Österreichische Reichsgeschichte. Geschichte der Staatsbildung und des öffentlichen Rechts. 2. erweiterte und verbesserte Auflage ... herausgegeben und bearbeitet von A. Dopsch. Wien, F. Tempsky 1901. XII, $372 \mathrm{~S}$.

Unsere Anzeige wird sich damit begnügen dürfen, kurz der Eigenart von A. Hubers "Österreichischer Reichsgeschichte" zu gedenken, um alsdann auf die Veränderungen einzugehen, die das Buch in seiner neuen Gestalt aufweist.

Das Werk des am 23. November 1898 verstorbenen Geschichtschreibers ist wie diejenigen von A. Bachmann, A. Luschin von Ebengreuth und E. Werunsky aus dem Bedürfnisse des akademischen Unterrichts hervorgegangen, nachden die Studienordnung für die juristischen Fakultäten vom Jahre 1893 die österreichische Reichsgeschichte für ein obligates Fach erklärt hatte. Nicht eine Geschichte des Rechts in seiner Totalität ist beabsichtigt; nur die Wandlungen der staatlichen Zusammensetzung Österreichs, die Entwicklung seiner Verfassung und Verwaltung sollen dargelegt werden, also nicht auch die Abfolge derjenigen Formen, in denen Privat-, Straf- und Prozeßrecht sich geüußert haben. Huber gliedert den Stoff in fünf Perioden: die erste reicht bis zum Jahre 1526 als dem der Vereinigung von Böhmen und Ungarn mit den österreichischen Ländern, die zweite bis 1740 , d. h. bis zum Erlöschen des Mannesstamms der Habsburger; die Reformen sodann unter Maria Theresia und Josef II., die Zustände im Zeitraum von 1792-1848 sind die Voraussetzungen für das öffentliche Recht der Gegenwart, bis an deren Schwelle selbst das Buch geführt ist. Die Betrachtung der Neuzeit also beansprucht den weitesten Raun, während das Mittelalter weit knapper bedacht ist: man weiß, wie summarisch in den deutschen Rechtsgeschichten die Jahrhunderte seit der Reformation

1) Die Besprechung ron Seeliger, Die soziale und politische Bedeutung der Grundherrschaft im frübern Mittelalter 1903 und einiger anderer Erscheinungen des laufenden Jahres konnte leider für das vorliegende Heft nicht mehr ermöglicht und mußte für das nächste verspart werden.

Für die Redaktion. U. St. 
behandelt sind, wie sehr sie die Würdigung andererseits der jüngsten Vergangenheit den Lehrbüchern des Staatsrechts überlassen, nicht ohne zugleich auf Darstellungen der partikularen Rechtsgeschichte bäufiger als vielleicht erwünscht zu verweisen. Huber legt weiterhin in allen Abschnitten den Hauptnachdruck auf die Schilderung der Staatsbildung, ihre mannigfachen Verschiebungen. Er breitet reiches Detail aus, das sich nicht immer sofort und leicht - ich spreche ja als Nichtösterreicher - dem Gedächtnis einprägen will. Damit aber hängt endlich zusammen, daß die Betrachtung des öffentlichen Rechts mehr den dauernden Flu $\beta$ seiner Entfaltung veranschaulicht als die durchgüngig, überall und immer wirksamen Tendenzen seines Werdens und Wirkens. Man könnte sagen, das Buch ist dank seiner Methode in erster Linie ein geschichtliches, in zweiter erst ein Hülfsmittel der rechtsgeschichtlichen Betrachtung. Dadurch gewinnt es an praktischer Verwendbarkeit im Einzelfalle, aber das Bedürfnis nach Zusammenfassung wird nicht ganz befriedigt; es vermittelt reiche Kenntnisse im einzelnen, nicht ein politisches Verständnis, politisch hier wie natürlich im ursprünglichen Wortsinne genommen.

Der Herausgeber, A. Dopsch, war an diese Eigenart, an die von A. Huber seiner Arbeit gezogenen Richtlinien gebunden. Es konnte nicht seine Aufgabe sein, daran zu ruitteln; er wollte ja kein neues Werk an die Stelle des einmal eingebürgerten setzen. Auf der auderen Seite mußte er es in Ubereinstimmung bringen mit den Ergebnissen der jüngsten Forschung, die seit dem Jahre 1895 - in ihm war die Vorlage zum ersten Male erschienen - nicht gefeiert hatte, zumal die Anregung des erwähnten Studienplanes von 1893 stetig fortwirkte. Schon Huber hatte eine Neubearbeitung ins Auge gefaßt; das Vorgehen von Dopsch ist vermutlich einschneidender als jener es beabsichtigt, aber es ist berechtigt. Nirgends ist die scharfe Grenzlinie überschritten $\mathrm{zwischen}$ der Pietät gegenüber dem Toten und der Forderung, das wirklich Veraltete durch Neues und gleichzeitig Besseres zu ersetzen. Drei Schichten solcher Änderungen lassen sich namhaft machen. Einmal Verschiebungen in der Disposition des Stoffes, sodann Zutaten, deren Aufgabe es ist, den überkommenen Text reichlicher auszufüllen, un durch "eine Skizzierung der die Entwicklung bestimmenden allgemeinen verfassungsgeschichtlichen Tendenzen die Erfassung des nackten Tatsachenmateriales anzubahnen ${ }^{\text {. }}$. So werden jetzt z. B. die Reformen Maximilians I. eingehender behandelt als zuvor (vgl. S. 87-91 der zweiten Auflage mit S. 63-66 der ersten); nicht minder zahlreich sind die Zusätze zur Charakteristik Josefs II.: sie alle einzufügen mochte nicht immer leichte Mühe sein, wenn anders der ursprüngliche Rahmen nicht ganz gesprengt werden sollte (vgl. S. 267-283 mit S. 205 bis 215 der ersten Auflage). An dritter Stelle endlich stehen mehrere gänzlich neue Abschnitte, so vor allem diejenigen über die Gesetzgebung in den österreichischen ländern während des Mittelalters (S. 82 ff.) und die Rechtsquellen vom Ausgang des Mittelalters bis 
zum Jahre 1740 (S. 160 ff.), die freilich stets ein Zurückgreifen auf die Arbeiten von Luschin (S. $29 \mathrm{ff}$., $345 \mathrm{ff}$.) oder Werunsky (S. $3 \mathrm{ff}$.) nötig machen werden, da sie zu wenig Gewicht legen auf die Namhaftmachung der Ausgaben und sonstiger Literatur. Dankenswert ist sodann die Eiureihung verschiedener Stammbäume: ihr Fehlen in der ersten Auflage hatte häufig die Lektüre erschwert, nicht zuletzt aus dem Grunde, weil in keinem anderen Staate die Schicksale der zusammenhaltenden Dynastie von so hoher Bedeutung waren als in dem überwiegend patrimonialen Österreich. $\mathrm{Ob}$ es sich aber nicht empfohlen hätte, die Stammbäume insgesamt in einen eigenen Anhang zu verweisen? Wie dem immer sei, das Maß der Zutaten, sei es nun Hubers, sei es derjenigen von Dopsch - mit Fug ist keine äußerlich kenntlich machende Unterscheidung durchgeführt und nur im allgemeinen gibt das Vorwort einige Fingerzeige -, die dadurch bedingte Vermehrung des Uwfangs (372 S. gegen 280 S. der ersten Auflage) hat den Charakter des handlichen Lehrbuchs nicht zerstört. Für eine dritte Auflage ist jedenfalls die Anfertigung eines Registers und die Beigabe von kartographischem Material (vgl. auch die durchsichtige Ländertafel bei Luschin S. 5) in Erwägung zu ziehen. Gerade bei der österreichischen Reichsgeschichte ist die Verbindung historischen Wissens mit geographischer Anschauung eine der ersten Voraussetzungen des Verständnisses. Bevor aber eine solche erscheint, werden die Bemühungen des Herausgebers dem Buche von A. Huber gesteigerte Verbreitung gewährleisten.

Greifswald.
A. Werminghoff.

A. M. Ehrentraut, Untersuchungen über die Frage der Frei- und Reichsstädte (Leipziger Studien aus dem Gebiet der Geschichte IX, 2). Leipzig, B. G. Teubner 1902. VIII, $172 \mathrm{~S} .8^{0}$.

Als Beitrag zur deutschen Verfassungsgeschichte des späteren Mittelalters wird die vorliegende Arbeit auch dem willkommen sein, der nicht gegen die Ergebnisse, wohl aber gegen den Aufbau der Untersuchung Einwände zu erheben hat. Ihr Thema ist die Beantwortung der Frage, welcher Art die Beziehungen der sieben gemeinhin als Freistädte bezeichneten Gemeinwesen (Mainz, Worms, Speier, Köln, Straßburg, Basel und Regensburg) zum Reichsoberhaupt waren, inwiefern sich diese von denen der übrigen Reichsstädte unterschieden. Vorauf geht ein Rückblick auf die ältere, vor allem die publizistische Literatur des siebzehnten und achtzehnten Jahrhunderts; als die Quelle der heute herrschenden Ansicht ergibt sich die Darlegung von A. Heusler (Ursprung der deutschen Stadtverfassung 1872, S. $238 \mathrm{ff}$.): seine scharfe Umgrenzung des Begriffs der Freistadt, die dem Bischof nicht als Landstadt zugehöre, dem König aber nur Heerdienst zu leisten habe zu 